\title{
Darstellung symmetrischer Functionen durch die Potenzsummen.
}

\author{
(Von Herrn Hermann Hankel in Leipzig.)
}

Bekanntlich hat Waring in seinen „meditationes algebraicae“ 1770 eine Formel gegeben, durch welche jede homogene, rationale ganze, symmetrische Function der Wurzeln $x_{1}, x_{2}, \ldots x_{m}$ einer algebraischen Gleichung als Function der Potenzsummen und zwar durch eine Summe von Producten solcher dargestellt wird. In einer viel übersichtlicheren, aber, wie es scheint, bisher noch nicht bemerkten Weise kann man dieselbe Aufgabe mit Hülfe von Determinanten lösen.

I. Es lässt sich nämlich jede symmetrische Function

$$
\left(\alpha_{1}, \alpha_{2}, \ldots \alpha_{n}\right)=\Sigma x_{1}^{\alpha_{1}} x_{2}^{\alpha_{2}} \ldots x_{n}^{\alpha_{n}}
$$

wo unter dem Summenzeichen alle möglichen Combinationen und Permutationen der Wurzeln $x_{1} \ldots x_{m}$ an Stelle von $x_{1} \ldots x_{*}$ zu setzen sind, durch eine Summe von Determinanten ausdrücken, welche aus

$$
\text { (1.) }\left|\begin{array}{llllc}
\boldsymbol{s}_{\alpha_{1}} & \mathbf{1} & \mathbf{0} & \ldots & 0 \\
\boldsymbol{s}_{\alpha_{1}+\alpha_{2}} & \boldsymbol{s}_{\alpha_{2}} & 2 & \ldots & 0 \\
\boldsymbol{s}_{\alpha_{1}+\alpha_{2}+\alpha_{3}} & \boldsymbol{s}_{\alpha_{2}+\alpha_{3}} & \boldsymbol{s}_{\alpha_{3}} & \ldots & 0 \\
\vdots & \vdots & \vdots & & \vdots \\
\vdots & \vdots & \vdots & & n-1 \\
\boldsymbol{s}_{\alpha_{1}+\alpha_{2}+\ldots+\alpha_{n}} & \boldsymbol{s}_{\alpha_{2}+\alpha_{3}+\ldots+\alpha_{n}} & \boldsymbol{s}_{\alpha_{3}+\ldots+\alpha_{n}} & \ldots & \boldsymbol{s}_{\alpha_{n}}
\end{array}\right|
$$

hervorgehen, wenn man die $\alpha_{1}, \ldots \alpha_{n}$ auf jede Weise permutirt; und zwar ist diese Summe:

$$
=n !\left(\alpha_{1}, \ldots \alpha_{n}\right) .
$$

Die wirkliche Darstellung gestaltet sich auf diese Weise keineswegs so complicirt, als es auf den ersten Blick scheinen könnte. So hat man z. B. um

$$
5 ! \Sigma x_{1}^{a} x_{2}^{b} x_{3}^{c} x_{4}^{d} x_{5}^{e}
$$

durch die Potenzsummen darzustellen, zunächst die Determinante: 


$$
\begin{aligned}
& \left|\begin{array}{lllll}
\boldsymbol{s}_{a} & \mathbf{1} & \mathbf{0} & \mathbf{0} & \mathbf{0} \\
\boldsymbol{s}_{a+b} & \boldsymbol{s}_{b} & \mathbf{2} & \mathbf{0} & \mathbf{0} \\
\boldsymbol{s}_{a+b+c} & \boldsymbol{s}_{b+c} & \boldsymbol{s}_{c} & \mathbf{3} & \mathbf{0} \\
\boldsymbol{s}_{a+b+c+d} & \boldsymbol{s}_{b+c+d} & \boldsymbol{s}_{c+d} & \boldsymbol{s}_{d} & \mathbf{4} \\
\boldsymbol{s}_{a+b+c+d+e} & \boldsymbol{s}_{b+c+d+e} & \boldsymbol{s}_{c+d+e} & \boldsymbol{s}_{d+e} & \boldsymbol{s}_{\boldsymbol{p}}
\end{array}\right| \\
& =s_{a} s_{b} s_{c} s_{d} s_{e}-\left\{4 s_{a} s_{b} s_{c} s_{d+e}+3 s_{a} s_{b} s_{e} s_{c+d}+2 s_{a} s_{d} s_{e} s_{b+c}+s_{c} s_{d} s_{e} s_{a+b}\right\} \\
& +\left\{12 s_{a} s_{b} s_{c+d+e}+6 s_{a} s_{e} s_{b+c+d}+2 s_{d} s_{e} s_{a+b+c}\right\} \\
& -\left\{24 s_{a} s_{b+c+d+e}+6 s_{e} s_{a+b+c+d}\right\}+\left\{8 s_{a} s_{b+c} s_{d+e}+4 s_{c} s_{a+b} s_{d+e}+3 s_{e} s_{a+b} s_{c+d}\right\} \\
& -\left\{12 s_{a+b} s_{c+d+e}+8 s_{d+e} s_{a+b+c}\right\}+24 s_{a+b+c+d+e}
\end{aligned}
$$

zu entwickeln. Dann hat man alle Permutationen der $a, b, c, d, e$ vorzunehmen und zu addiren; dabei wird z. B. aus $s_{a} s_{b} s_{e} s_{c+d}$ jedes Product dieser Form also $s_{a} s_{b} s_{c} s_{d+e}$ nur einmal erzeugt, wenn zunächst die Vertauschbarkeit der Factoren $s_{a}, s_{b}, s_{c}$ und der Summanden $d$ und $e$ in $s_{d+e}$ ignorirt wird. Ebenso wird aus $s_{a} s_{d} s_{e} s_{b+c}$ und $s_{c} s_{d} s_{e} s_{a+b}$ in diesem Sinne das Product $s_{a} s_{b} s_{c} s_{d+e}$ nur einmal erhalten. In der Summe wird dasselhe sonach mit dem Factor $4+3+2+1=10$ erscheinen, und so überhaupt:

$$
\begin{aligned}
5 !(a, b, c, d, e)= & \sum\left\{s_{a} s_{b} s_{c} s_{d} s_{e}-10 s_{\alpha} s_{b} s_{c} s_{d+e}+20 s_{a} s_{b} s_{c+d+e}\right. \\
& \left.-30 s_{a} s_{b+c+d+e}+15 s_{a} s_{b+c} s_{d+e}-20 s_{a+b} s_{c+d+e}+24 s_{a+b+c+d+e}\right\},
\end{aligned}
$$

wo in dieser Summe alle möglichen Permutationen zu bilden sind. Da aber die sämmtlichen so entstehenden Glieder von der Form $s_{a} s_{b} s_{c} s_{d} s_{e}$ identisch sind, so ist $\Sigma s_{l i} s_{b} s_{c} s_{d} s_{e}=\mathbf{5} ! s_{a} s_{b} s_{c} s_{d} s_{e}$. Da ferner die Permutationen von $a, b, c$ einerseits und $c, d$ andererseits, das Product $s_{a} s_{b} s_{c} s_{d+e}$ in Wahrheit nicht ändern, so wird dasselbe $3 ! 2 !$ mal in der obigen Summe erscheinen und so $\Sigma s_{a} s_{b} s_{c} s_{d+e}=12 S s_{a} s_{b} s_{c} s_{d+e}$ sein, wenn unter $S$ nur die Summe der wirklich verschiedenen Producte verstanden wird. Ebenso wird jedes Product $s_{a} s_{b} s_{c+d+e}$ in obiger Summe $2 ! 3 !$ mal gebildet, $s_{a} s_{b+c+d+e} 4 ! \mathrm{mal}$; in $\boldsymbol{s}_{a} \boldsymbol{s}_{b+c} \boldsymbol{s}_{d+e}$ wird man sowohl $b$ und $c$ als auch $d$ und $e$, sowie auch überdies diese beiden Gruppen $b+c$ und $d+e$ mit einander vertauschen können, ohne dass ein anderes Product erhalten wird; dasselbe wird daher $2 ! 2 ! 2 !$ mal in der obigen Summe erzeugt. Ferner erhält man $s_{a+b} s_{c+d+e} 2 ! 3 !$ mal und endlich $s_{a+b+c+d+e}$ 5! mal, so dass man:

$$
\begin{aligned}
(a, b, c, d, e) & =s_{a} s_{b} s_{c} s_{d} s_{e}-\oint s_{a} s_{b} s_{c} s_{d+e}+2 \oint s_{a} s_{b} s_{c+d+e} \\
& -6 \oint s_{a} s_{b+c+d+e}+\delta s_{a} s_{b+c} s_{d+e}-2 \oint s_{a+b} s_{c+d+e}+24 s_{a+b+c+d+e}
\end{aligned}
$$


findet, wo sich die Summen überall auf die Bildung aller wirklich verschiedenen Glieder derselben Form beziehen.

Ein besonderer Fall unserer allgemeinen Formel ist schon bekannt: Wenn nämlich alle Exponenten $\alpha_{1}, \ldots \alpha_{n}=1$ und damit auch alle Determinanten der obigen Summe einander gleich sind, so erhält man:

$$
\sum x_{1} x_{2} \ldots x_{n}=\left|\begin{array}{cccccc}
s_{1} & 1 & 0 & \ldots & 0 \\
s_{2} & s_{1} & 2 & . & 0 \\
\vdots & \vdots & \vdots & & \vdots \\
s_{n} & s_{n-1} & s_{n-2} & . & . & s_{1}
\end{array}\right| .
$$

Diese Summe ist aber nicht unmittelbar $\operatorname{der} n^{\text {1e }}$ Coefficient der algebraischen Gleichung, deren Wurzeln $x_{1}, x_{2}, \ldots x_{m}$ sind, sondern das $n$ ! fache desselben; denn in $\operatorname{der} \Sigma$ hat man nicht allein alle Combinationen zu je $n$, sondern auch alle Permutationen innerhalb einer jeden Combination $x_{1}, x_{2}, \ldots x_{n}$ eintreten zu lassen. Auf diesen Umstand hat man überhaupt zu achten, wenn man eine symmetrische Function $\left(\alpha_{1}, \ldots \alpha_{n}\right)$, in der nicht alle Exponenten von einander verschieden sind, durch die Summe von Determinanten darstellen will.

II. Um den obigen Satz I. nun allgemein zu erweisen, gehe ich von einer anderen Form desselben aus. Ich werde nämlich zeigen:

Dass $(n-1) !\left(\alpha_{1}, \ldots \alpha_{n}\right)$ durch eine Summe von $(n-1)$ ! Determinanten von der Form (1.) dargestellt wird, welche man durch Permutation der Indices $\alpha_{1}, \ldots \alpha_{n-1}$ erhält, während der letzte Index $\alpha_{n}$ und damit die letzte Verticalreihe, in den Determinanten überall unverändert bleibt.

Dass hiermit zugleich die obige Form I. des Satzes erwiesen wird, ist evident, denn man braucht, um diese zu erhalten, nur in jeder Determinante noch die Permutationen von $\alpha_{n}$ mit $\alpha_{1}, \ldots \alpha_{n-1}$ vorzunehmen.

Was aber die jetzige Form betrifft, so kann dieselbe für $n=2,3$ leicht verificirt werden; denn nach ihr ist:

$$
\begin{gathered}
\Sigma x_{1}^{\alpha_{1}} x_{2}^{\alpha_{2}}=\left|\begin{array}{ll}
s_{a_{1}} & 1 \\
s_{\alpha_{1}+\alpha_{2}} & s_{\alpha_{2}}
\end{array}\right|=s_{\alpha_{1}} s_{\alpha_{2}}-s_{\alpha_{1}+\alpha_{2}}, \\
2 ! \Sigma x_{1}^{\alpha} x_{2}^{\alpha_{2}} x_{3}^{\alpha_{3}}=\left|\begin{array}{lll}
s_{\alpha_{1}} & 1 & 0 \\
s_{\alpha_{1}+\alpha_{2}} & s_{\alpha_{2}} & 2 \\
s_{\alpha_{1}+\alpha_{2}+\alpha_{3}} & s_{\alpha_{2}+\alpha_{3}} & s_{\alpha_{3}}
\end{array}\right|+\left|\begin{array}{lll}
s_{\alpha_{2}} & 1 & 0 \\
s_{\alpha_{2}+\alpha_{1}} & s_{\alpha_{1}} & 2 \\
s_{\alpha_{2}+\alpha_{1}+\alpha_{3}} & s_{\alpha_{1}+\alpha_{3}} & s_{\alpha_{3}}
\end{array}\right| \\
=2\left\{s_{\alpha_{1}} s_{\alpha_{2}} s_{\alpha_{3}}-s_{\alpha_{1}} s_{\alpha_{2}+\alpha_{3}}-s_{\alpha_{2}} s_{\alpha_{3}+\alpha_{1}}-s_{\alpha_{3}} s_{\alpha_{1}+\alpha_{2}}+2 s_{\alpha_{1}+\alpha_{2}+\alpha_{3}}\right\} .
\end{gathered}
$$

Durch eine bekannte Schlussweise kann man hieraus die Formel allgemein 
erweisen mittels des Satzes:

$$
\left(\alpha_{1}, \ldots \alpha_{n}\right)=s_{\alpha_{i_{n}}}\left(\alpha_{i_{1}}, \ldots \alpha_{i_{n-1}}\right)-\Sigma\left(\alpha_{i_{1}}, \ldots \alpha_{i_{n-2}}, \alpha_{i_{n-1}}+\alpha_{i_{n}}\right)
$$

worin $i_{n}$ eine beliebige, aber bestimmte Zahl aus der Reihe 1, 2, . n bezeichnet, und $i_{n-1}$ in der $\Sigma$ folgeweise die Werthe $1,2, \ldots n$, mit Ausschluss der für $i_{n}$ gewählten Zahl zu durchlaufen hat, die Zahlen $i_{1}, \ldots i_{n-2}$ aber die übrigen $(n-2)$ Zahlen aus $1,2, \ldots n$ in irgend einer Reihenfolge bezeichnen.

Setzt man nun voraus, dass der Satz in der II. Form für eine homogene Function $(n-1)^{\text {ter }}$ Ordnung erwiesen ist, so hat man danach

$$
(n-2) !\left(\alpha_{i_{1}}, \ldots \alpha_{i_{n-2}}, \alpha_{i_{n-1}}+\alpha_{i_{n}}\right)=
$$

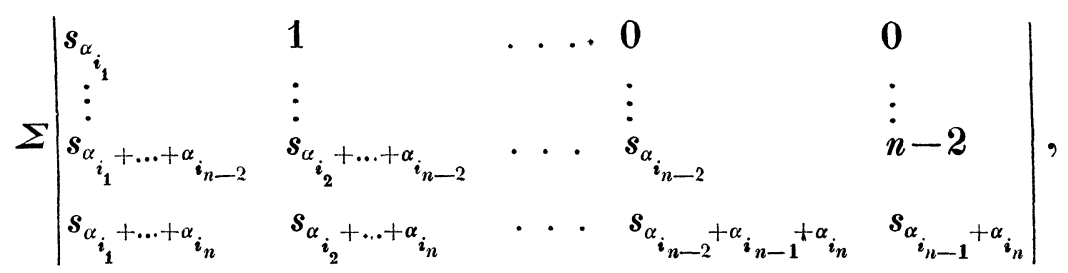

wo in dieser Summe $i_{1}, \ldots i_{n-2}$ alle möglichen Permutationen der Zahlenwerthe $1,2, \ldots n$, mit Ausschluss der für $i_{n-1}$ und $i_{n}$ gewählten, zu durchlaufen haben. Durch Hinzufügung einer neuen vertikalen und horizontalen Reihe kann man für dieselbe Summe:

$$
-\frac{1}{n-1} \sum\left|\begin{array}{lllll}
s_{\alpha_{i_{1}}} & \ldots & 0 & 0 & 0 \\
\vdots & & \vdots & \vdots & \vdots \\
s_{\alpha_{i_{1}}+\ldots+\alpha_{i_{n-2}}} & \ldots & s_{\alpha_{i_{n-2}}} & n-2 & 0 \\
s_{\alpha_{i_{1}}+\ldots+\alpha_{i_{n-1}}} & \ldots & s_{\alpha_{i_{n-2}}+\alpha_{i_{n-1}}} & s_{\alpha_{i_{n-1}}} & n-1 \\
s_{\alpha_{i_{1}}+\ldots+\alpha_{i_{n}}} & \ldots & s_{\alpha_{i_{n-2}}+\alpha_{i_{n-1}}+\alpha_{i_{n}}} & s_{\alpha_{i_{n-1}}+\alpha_{i_{n}}} & 0
\end{array}\right|
$$

schreiben, und man sieht daher, dass

$$
-\Sigma\left(\alpha_{i_{1}}, \ldots \alpha_{i_{n-2}}, \alpha_{i_{n-1}}+\alpha_{i_{n}}\right),
$$

wo die Summe in dem oben bei (2.) angegebenen Sinne zu nehmen ist, durch

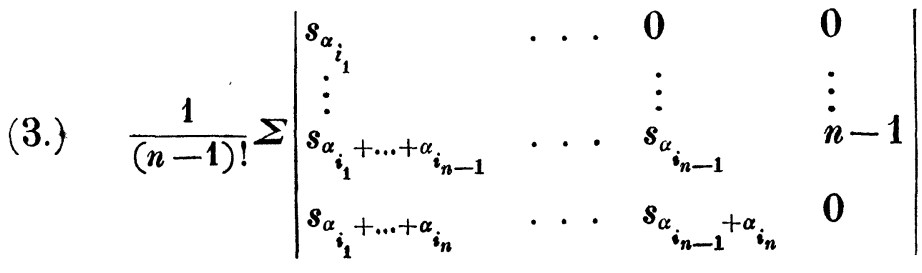


dargestellt wird, in welcher Summe jetzt $i_{1}, \ldots i_{n-2}$ and $i_{n-1}$ alle Permutationen in der Zahlenreihe $1,2, \ldots n$ mit Ausschluss des für $i_{n}$ gewählten Werthes zu durchlaufen haben.

Gilt ferner unser Satz II. für eine Function $(n-1)^{\text {ter }}$ Ordnung, so gilt, wie schon bemerkt, für eben diese auch der Satz I., es ist also:

$$
\left(\alpha_{i_{1}}, \ldots \alpha_{i_{n-1}}\right)=\frac{1}{(n-1) !} \Sigma\left|\begin{array}{lll}
s_{\alpha_{i_{1}}} & \cdots & 0 \\
\vdots & & \vdots \\
s_{\alpha_{i_{1}}+\ldots+\alpha_{i_{n-1}}} & \cdots & s_{\alpha_{i_{n-1}}}
\end{array}\right|,
$$

oder durch Hinzufügung einer horizontalen und einer vertikalen Reihe:

(4.)

$$
s_{\alpha_{i_{n}}}\left(\alpha_{i_{1}}, \ldots \alpha_{i_{n-1}}\right)=\frac{1}{(n-1) !} \Sigma\left|\begin{array}{llll}
s_{\alpha_{i_{1}}} & \cdots & 0 & 0 \\
\vdots & & \vdots & \vdots \\
s_{\alpha_{i_{1}}+\ldots+\alpha_{i_{n-1}}} & \cdots & s_{\alpha_{i_{n-1}}} & 0 \\
s_{\alpha_{i_{1}}+\ldots+\alpha_{i_{n}}} & \cdots & s_{\alpha_{i_{n-1}}+\alpha_{i_{n}}} & s_{\alpha_{i_{n}}}
\end{array}\right|,
$$

wo das Summenzeichen sich auf dieselben Permutationen bezieht, wie in (3.). Die Determinanten in (3.) und (4.) unterscheiden sich nur in der letzten Columne, können also sofort addirt werden und man erhält so nach (2.):

$$
\left(\alpha_{1}, \ldots \alpha_{n}\right)=\frac{1}{(n-1) !} \Sigma\left|\begin{array}{llc}
s_{\alpha_{i_{1}}} & \ldots & 0 \\
\vdots & & \vdots \\
s_{\alpha_{i_{1}}+\ldots+\alpha_{i_{n}}} & \ldots & \boldsymbol{s}_{\alpha_{i_{n}}}
\end{array}\right|,
$$

worin $i_{n}$ irgend eine der Zahlen $1,2, \ldots n$ bedeutet und $i_{1}, \ldots i_{n-1}$ die Permutationen der übrig bleibenden Zahlen zu durchlaufen haben, q. e. d.

Leipzig, April 1865. 